\title{
Latex on glass: an appropriate model for cartilage-lubrication studies?
}

\section{Journal Article}

Author(s):

Roba, Marcella; Bruhin, Christoph; Ebneter, Urs; Ehrbar, Roman; Crockett, Rowena; Spencer, Nicholas D.

Publication date:

2010-06

Permanent link:

https://doi.org/10.3929/ethz-b-000413193

Rights / license:

In Copyright - Non-Commercial Use Permitted

Originally published in:

Tribology Letters 38(3), https://doi.org/10.1007/s11249-010-9603-7 


\title{
Latex on Glass: an Appropriate Model for Cartilage-Lubrication Studies?
}

\author{
Marcella Roba $\cdot$ Christoph Bruhin . \\ Urs Ebneter • Roman Ehrbar · Rowena Crockett • \\ Nicholas D. Spencer
}

Received: 17 February 2010/Accepted: 30 March 2010/Published online: 17 April 2010

(C) Springer Science+Business Media, LLC 2010

\begin{abstract}
Latex versus glass has frequently been used as a model system for the investigation of natural lubrication mechanisms, despite its significant differences from articular cartilage pairings. The differences in surface chemistry account for its different behavior in terms of protein adsorption and lubrication. While cartilage is well known for its protein resistance, most proteins present in synovial fluid can non-specifically adsorb onto latex or glass. We have investigated latex-versus-glass lubrication by means of pin-on-disk tribometry in the presence of synovial-fluid proteins and glycoproteins, focusing on the influence of the glass-cleaning procedure on friction. In order to simulate the effects of possible contamination of glass in previous studies, both hydrophilic and hydrophobic glass substrates were tested. Albumin was shown to impair lubrication (in comparison to PBS) when latex was slid against both types of glass surface, whereas bovine synovial fluid (BSF) and alpha-1-acid glycoprotein (AGP) impaired the lubrication of latex versus hydrophilic glass and improved the lubrication of latex versus hydrophobic glass. Protein adsorption on the surfaces was monitored by means of fluorescence imaging and optical waveguide lightmode spectroscopy (OWLS), which revealed a faster and greater amount of adsorption of AGP on hydrophobic surfaces than
\end{abstract}

\footnotetext{
M. Roba $\cdot$ R. Crockett

Empa, Swiss Federal Institute for Material Science and Technology, Ueberlandstrasse 129, 8600 Dübendorf, Switzerland

M. Roba · C. Bruhin - U. Ebneter · R. Ehrbar ·

N. D. Spencer $(\square)$

Laboratory for Surface Science and Technology, Department of Materials, ETH Zurich, Wolfgang-Pauli-Strasse 10, 8093 Zurich, Switzerland

e-mail: spencer@mat.ethz.ch
}

on hydrophilic ones. The influence of surface chemistry on the friction behavior of BSF and on the adsorption of AGP suggests that it plays a role in determining the relative amounts of adsorbed synovial fluid proteins. When BSF is used as a lubricant in the latex-versus-hydrophobic-glass system, more of the AGP, relative to albumin, appears to adsorb on both surfaces, counteracting the negative effect of albumin on friction. It therefore seems that latex on glass, while displaying nominal similarities to cartilage on cartilage under certain conditions, is not a useful model system. Moreover, surface contamination of the glass can play a major role in determining the results.

Keywords Glass - Latex - Cartilage $\cdot$ Lubrication · Biotribology

\section{Introduction}

Under physiological conditions, healthy articular joints exhibit excellent tribological properties, thanks to the combination of synovial fluid and articular cartilage [1]. Friction coefficients have been shown to be very low, in the range of $0.0005-0.04[2,3]$, although the exact mechanism of lubrication is not yet fully understood [4]. Lubricin, a glycoprotein present in synovial fluid [5], has been suggested to be responsible for the boundary lubrication of articular joints, i.e. when they are subjected to high loads and low speeds $[6,7]$. In tests used to demonstrate this superior lubricating ability, a set-up was used in which cartilage slid against itself [8] or against polished glass [6]. It was shown that the friction of systems lubricated by lubricin or by synovial fluid was similar, leading to the bold conclusion that lubricin is responsible for the lubricating properties of synovial fluid. The use of cartilage 
surfaces in friction tests is not trivial, since the deformation of the cartilage itself leads to variations in the contact area and pressure, and is difficult to control. In addition, the mechanical properties of cartilage depend on the location from which it is harvested and tend to be affected by the environment during removal from the joint, making reproducibility in the friction tests very difficult to achieve $[9,10]$. Therefore, a well-defined model system that is capable of mimicking natural cartilage would be highly desirable. In many studies, latex versus glass has been used as such a model. Reciprocating sliding experiments carried out in the boundary regime showed that this tribopair gave similar friction coefficients for both lubricin and synovial fluid, and for this reason, it was thought to be capable of mimicking the cartilage-versus-cartilage system [11-14]. The lubricating properties of synovial fluid biomolecules other than lubricin have not been investigated with the latex-versus-glass apparatus.

In addition to macro-tribological studies, the role of lubricin in natural joint lubrication has been assessed by evaluating adsorption and interaction with model surfaces $[15,16]$. By means of atomic force microscopy (AFM), the surface forces apparatus (SFA), and surface plasmon resonance (SPR), lubricin was shown to adsorb on both hydrophilic and hydrophobic surfaces, forming brush-like layers with the sugar residues extending into solution together with loops and tails from the peptide chain. This was the basis for the suggestion that lubricin has both antiadhesive and chondroprotectant effects on cartilage surfaces, thus preventing wear [15]. The brush-like conformation has also been suggested to be responsible for the good lubricating properties of lubricin, similar to the mechanism of lubrication by charged polymers [17].

One of the most obvious differences between cartilage and glass/latex is the lack of resistance to non-specific protein adsorption in the latter, i.e. there is either a very selective or no adsorption of proteins on the cartilage surface. It is widely agreed that the most abundant protein in synovial fluid, albumin, does not play a role in the lubrication of natural joints. However, this protein will adsorb onto glass and latex and influence friction between these materials. We have investigated the lubrication of latex versus glass with bovine synovial fluid (BSF) to clarify whether indeed it is a useful model system, and also whether a single component, such as lubricin, could determine the friction in this system.

In particular, the influence of the procedure used to clean the glass substrates on protein adsorption and friction was investigated, since cleaning procedures may influence surface chemistry. Experiments were carried out using Piranha-cleaned glass slides, which resulted in highly hydrophilic surfaces, as well as ethanol-cleaned glass (as has frequently been used by others) and functionalized, hydrophobic glass, all sliding against latex. Protein adsorption on the surfaces (and close analogs) was also studied by means of both fluorescence imaging and optical waveguide lightmode spectroscopy (OWLS).

\section{Materials and Methods}

Additive-free latex (Interlatex $\mathrm{GmbH}$, Germany) was used to coat 9.5-mm-diameter cylindrical CoCrMo pins with one end shaped as a spherical section of radius $1,200 \mathrm{~mm}$ (Carpenter Technology Co. (USA), forged according to ISO 5832-12). The latex coating was approximately $80 \mu \mathrm{m}$ thick. The pins were rinsed with pure water and dried with nitrogen before being used for friction tests. For the proteinincubation tests, the same sample preparation procedure was applied using flat-ended CoCrMo cylinders. SuperFrost ${ }^{\circledR}$ Microscope Glass slides (Menzel-Gläser, Germany) were cleaned with Piranha solution (conc. sulfuric acid (70\%), hydrogen peroxide (30\%)), and subsequently boiled in pure water to eliminate sulfur residues. Hydrophilicity of the glass was determined by means of contact-angle measurements; the static contact angle was not measurably higher than zero. Hydrophobic glass was obtained via functionalization of piranha-cleaned slides with $1 \mathrm{H}, 1 \mathrm{H}, 2 \mathrm{H}, 2 \mathrm{H}$-perfluorooctyltrichlorosilanes (ABCR $\mathrm{GmbH} \& \mathrm{Co}$. KG, Germany) from the gas phase by 1-h exposure at room temperature in a desiccator. The resulting static contact angle was $100^{\circ}$. In previous, related studies, glass slides were reported to be cleaned by means of an ethanol-based detergent $[5,14]$. In order to mimic this treatment, slides were sonicated in pure ethanol for $10 \mathrm{~min}$. These ethanolcleaned glass slides displayed a static contact angle of $60^{\circ}$.

Bovine synovial fluid (BSF) was removed from the knee joint of cows no older than 18 months at the Hinwil slaughterhouse in Switzerland. All samples were clear, yellow, particle-free liquids, and were stabilized with protease inhibitor (Sigma-Aldrich, Switzerland) and 0.1\% sodium azide.

\subsection{Labeling of Proteins}

Fluorescence labeling of albumin and alpha-1-acid glycoprotein (AGP) was carried out with ATTO 488 NHS ester (Sigma-Aldrich, Switzerland). The structure of ATTO 488 is the undisclosed property of Atto-Tec $\mathrm{GmbH}$, Siegen, Germany (http://www.atto-tec.com).

Fatty-acid-free bovine serum albumin (BSA, SigmaAldrich, Switzerland) was dissolved in a sodium bicarbonate buffer $(0.1 \mathrm{M}, \mathrm{pH} 8.3$, Merck, Switzerland) to a concentration of $5 \mathrm{mg} / \mathrm{mL}$. ATTO NHS ester $(2 \mathrm{mg} / \mathrm{mL})$ was dissolved in amine-free, anhydrous dimethylsulfoxide (DMSO, Sigma-Aldrich, Switzerland) immediately before 
conjugation. The conjugation reaction was carried out by adding a twofold molar excess of dye to the protein solution followed by incubation for $60 \mathrm{~min}$ at $24^{\circ} \mathrm{C}$ with stirring. Labeled proteins were separated from the unreacted dye by dialysis overnight against phosphate-buffered saline (PBS, Sigma-Aldrich, Switzerland) using $10 \mathrm{kDa}$ dialysis membranes (Slide-A-Lyzer, Perbio Science N. V., Belgium). Aliquots of labeled albumin were stored at $-20^{\circ} \mathrm{C}$. The same protocol was used to label AGP (from bovine serum, Sigma-Aldrich, Switzerland).

\subsection{Adsorption Studies via Fluorescence Microscopy and OWLS}

Fluorescently labeled AGP and BSA were diluted in PBS to a concentration of $0.3 \mathrm{mg} / \mathrm{ml}$. A droplet of each solution was placed on latex, Piranha-cleaned glass, and silanefunctionalized glass samples, and the labeled proteins were allowed to adsorb for $60 \mathrm{~min}$ in a dark environment. After rinsing with PBS and pure water, fluorescence images at the border of the droplets were obtained with an AX10 Imager M1 m (Zeiss, Germany). The protein solutions were also used to incubate glass slides that had been cleaned at one end with ethanol and at the other with Piranha solution. This was to determine whether a contrast could be observed in protein adsorption according to surface wettability.

The BSA and AGP adsorptions were evaluated on hydrophilic (static contact angle close to $0^{\circ}$ ) and hydrophobic (static contact angle $100^{\circ}$ ) $\mathrm{SiO}_{2} / \mathrm{TiO}_{2}$ waveguides $\left(\mathrm{Si}_{0.75} \mathrm{Ti}_{0.25} \mathrm{O}_{2}\right.$ on glass, $1.2 \times 0.8 \mathrm{~cm}^{2}$, Microvacuum, Budapest, Hungary). Hydrophilic waveguides were obtained by rinsing with $0.1 \% \mathrm{HCl}$ and isopropanol, and subsequently cleaning with $\mathrm{O}_{2}$ plasma (Plasma Cleaner/ Sterilizer, PDC-32G instrument, Harrick, Ossining, NY, USA) for 2 min. To obtain hydrophobic surfaces, the waveguides were additionally functionalized with perfluorosilanes from the gas phase, as described previously. The static water contact angle of the functionalized waveguides was determined to be $100^{\circ}$.

Adsorption in the OWLS instrument (OWLS 110, Microvacuum, Hungary) was carried out at $37^{\circ} \mathrm{C}$, using PBS as a buffer. After having recorded the baseline, $0.5 \mathrm{mg} / \mathrm{ml}$ of BSA or AGP solution were injected into the flow chamber. Protein adsorption was allowed to proceed until a plateau in the adsorption curve was observed. At this stage, non- or weakly adsorbed proteins were washed away by rinsing the flow chamber with PBS.

\subsection{Pin-on-Disk Tribological Measurements}

Friction in the boundary regime was investigated by means of a pin-on-disk apparatus (CSM S.A., Switzerland).
During each experiment, the latex-coated CoCrMo pins were slid against Piranha-cleaned, ethanol-cleaned or silane-functionalized glass at a load of $1 \mathrm{~N}$ and a sliding speed of $10 \mathrm{~mm} / \mathrm{s}$ for 30 laps, using PBS as lubricant. At the 30th lap, AGP, BSA or BSF were added to the PBS, and sliding continued for another 30 laps. The sliding distance was $226 \mathrm{~cm}$. The final concentrations of AGP and BSA were 0.3 or $12.5 \mathrm{mg} / \mathrm{ml}$, respectively. By adding BSF to the PBS, the BSF was diluted by $50 \%$. At least three tests were carried out in each lubricant.

\section{Results}

3.1 Protein-Adsorption Analysis with Fluorescence Imaging and OWLS

Latex, Piranha-cleaned glass, and silane-functionalized glass samples were incubated in labeled AGP and BSA solutions. Both proteins adsorbed onto all three substrates (Fig. 1), as shown by the clear difference in the fluorescence intensity at the border of the droplets, indicating a difference between the blank and the area where protein adsorption had occurred.

It was also possible to observe changes in AGP adsorption qualitatively, according to the wettability of the surface (Fig. 2a). The fluorescence signal from adsorbed AGP was more intense on the ethanol-cleaned area of the glass (static contact angle $60^{\circ}$ ) than on the Piranha-cleaned region (static contact angle approximately $0^{\circ}$ ). The fluorescence following adsorption of labeled BSA was not visually different on the two areas (Fig. 2b).

Quantitative adsorption measurements carried out with OWLS showed that the amounts of BSA and AGP adsorbed onto a hydrophobic surface were $172 \pm 12$ and $110 \pm 14 \mathrm{ng} / \mathrm{cm}^{2}$, respectively. BSA adsorption on the hydrophilic surface was found to be $103 \pm 23 \mathrm{ng} / \mathrm{cm}^{2}$, whereas AGP adsorption was very low, and could not be quantified due to the absence of a stable plateau (Fig. 3).

\subsection{Pin-on-Disk Tribological Measurements}

The lubricating properties of solutions of AGP, BSA, and BSF in the latex-versus-glass system were investigated with a pin-on-disk tribometer. Figure 4 shows the friction curve for latex sliding against ethanol-cleaned glass immersed in BSF. The initial friction coefficient for PBS is very high and decreases after addition of BSF to the PBS.

The latex-versus-Piranha-cleaned-glass system was poorly lubricated by AGP in comparison to PBS (Fig. 5a). An increase in friction was also observed upon addition of BSA, and the degree of impairment was higher than with 
Fig. 1 Fluorescence images of latex samples after incubation with a labeled AGP and $\mathbf{b}$ labeled BSA, of Piranhacleaned glass after incubation with $\mathbf{c}$ labeled AGP and $\mathbf{d}$ labeled BSA, and of silanefunctionalized glass after incubation with e labeled AGP and $\mathbf{f}$ labeled BSA. Only part of the samples were incubated to allow a contrast with the blank surface
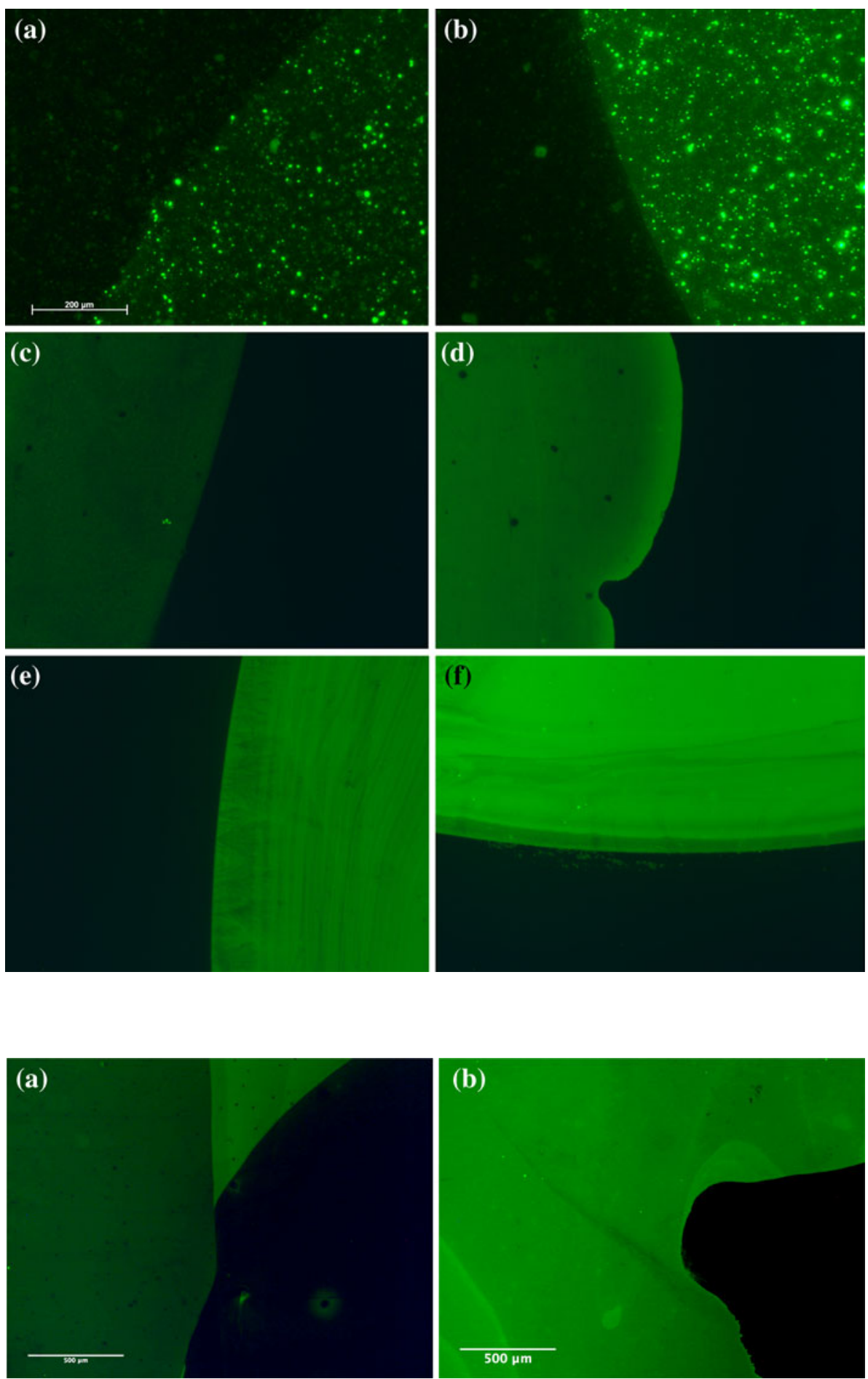

Fig. 2 Fluorescence images of glass samples half Piranhacleaned (left side) and half ethanol-cleaned (right side) after incubation with a labeled AGP and b labeled BSA. A blank area where no incubation occurred is present in both samples
AGP (Fig. 5b). When adding BSF to the PBS control, an increase in friction was also observed (Fig. 5c).

The addition of AGP to latex sliding against hydrophobized glass in PBS resulted in a decrease in friction (Fig. 6a), while BSA caused an increase in friction (Fig. 6b). Upon addition of BSF, similarly to AGP, an improvement in lubrication could be observed (Fig. 6c).

\section{Discussion}

The use of a well-defined model system for the investigation of natural joint lubrication is desirable, due to difficulties in controlling tribological experiments that involve cartilage as a sliding surface. The motivation in the literature for the choice of latex and glass as a model system 


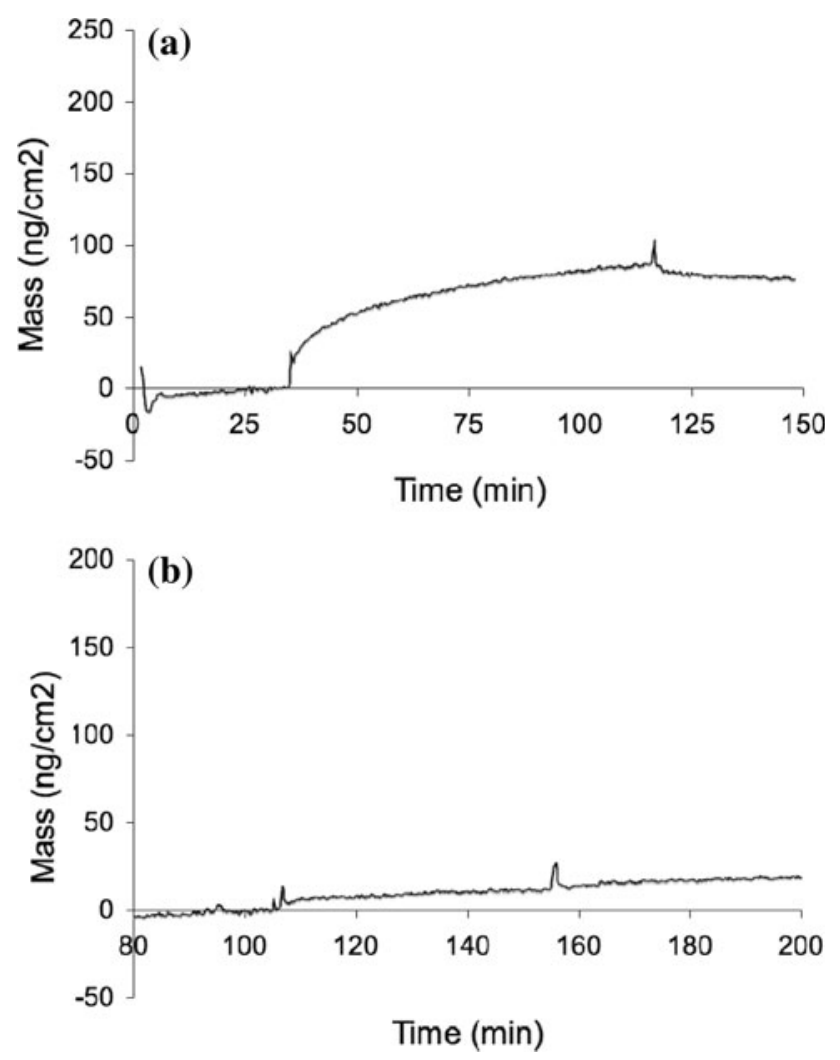

Fig. 3 OWLS curves for the adsorption of a BSA and b AGP on a hydrophilic surface

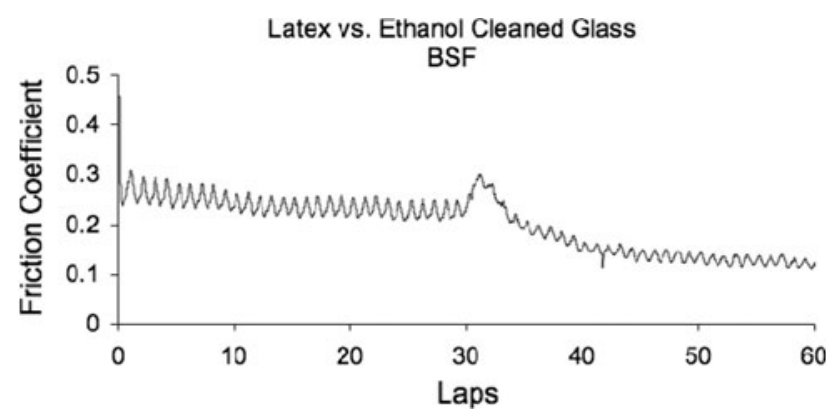

Fig. 4 Friction coefficients for latex sliding against ethanol-cleaned glass in PBS with addition of BSF at the 30th lap

was empirical, following the supposition that lubricin was responsible for synovial fluid lubricating ability [7]. As similar friction coefficients for both solutions of lubricin and synovial fluid had been observed, latex versus glass was thought to be a system capable of mimicking natural articular cartilage [11-14]. Moreover, latex on glass was shown to be lubricated by lubricin as efficiently as other tribological systems involving cartilage as one bearing surface, apparently validating the hypothesis [6]. The mechanical properties, surface chemistry, and resistance to protein adsorption of latex and glass, however, are quite different from those of cartilage.
Latex vs. Piranha cleaned glass

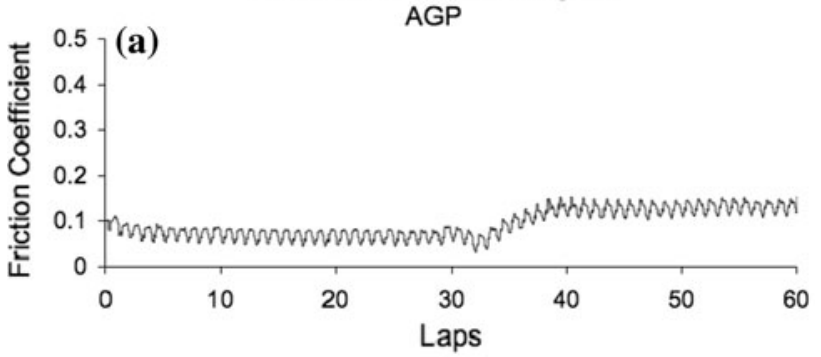

Latex vs. Piranha cleaned glass

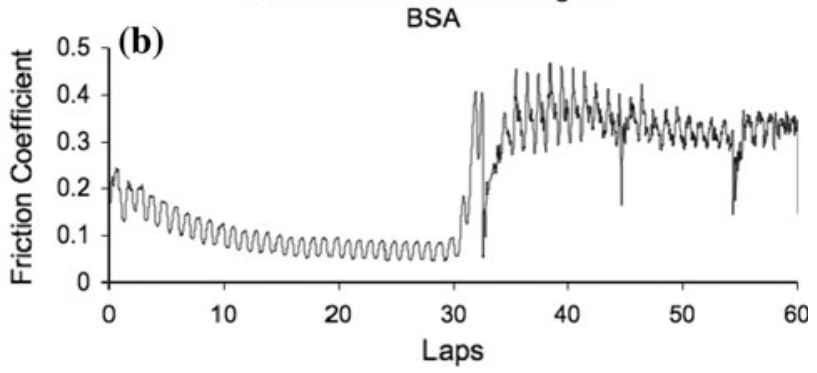

Latex vs. Piranha cleaned glass BSF

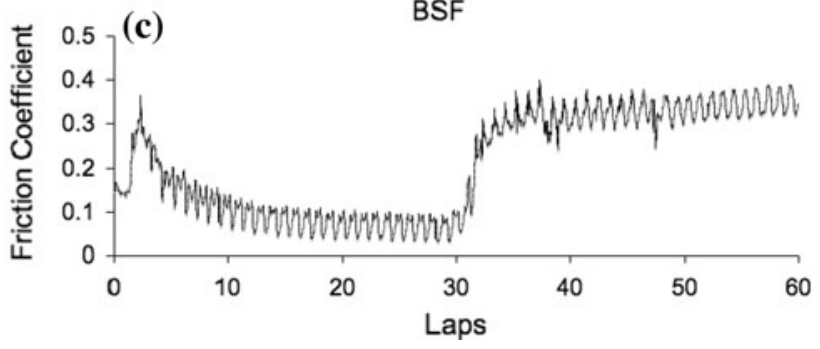

Fig. 5 Friction coefficients for latex sliding against Piranha-cleaned glass in PBS with addition of a AGP, $\mathbf{b}$ BSA, and $\mathbf{c}$ BSF at the 30th lap

In the case of latex and glass, the fluorescence imaging showed that both albumin and AGP adsorb from synovial fluid onto the surfaces. Therefore, it cannot be assumed that lubricin is the only protein adsorbing onto the surfaces, and it is highly unlikely to be the only protein that will influence friction. This lack of specific adsorption behavior was shown in a previous study by means of adsorption measurements on ultra-high-molecular-weight polyethylene (UHMWPE) and alumina [18], which are frequently used in joint prostheses. As fluorescence and SDS PAGE measurements have shown, a wide range of synovial-fluid proteins can adsorb onto a hydrophobic surface such as UHMWPE or latex. Other synovial fluid proteins can, therefore, adsorb and contribute to the friction of latex versus glass.

The lubricating behavior of other synovial fluid proteins was investigated. The setup used involved unidirectional sliding and constant load, which differs from the conditions in articular joints. This is inevitably a limitation, but this 

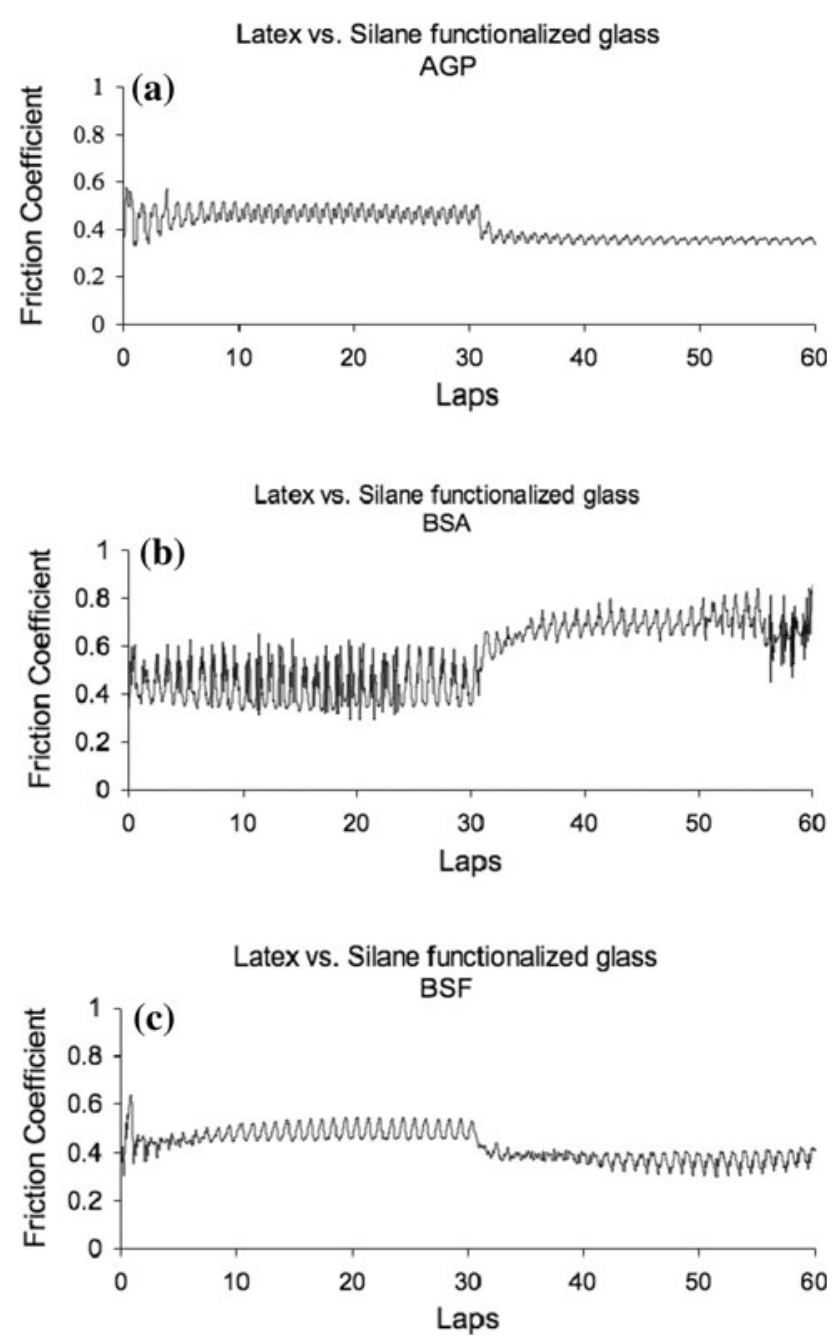

Fig. 6 Friction coefficients for latex sliding against silane-functionalized glass in PBS with addition of $\mathbf{a}$ AGP, $\mathbf{b}$ BSA, and $\mathbf{c}$ BSF at the 30th lap

work focused on the investigation of the role of surface chemistry in latex-on-glass lubrication, and this could be assessed by means of pin-on-disk tribometry. Albumin was chosen because it is the most abundant protein in synovial fluid and AGP because of its good lubricating properties [18]. Pin-on-disk experiments carried out using hydrophilic, Piranha-cleaned glass slides led to unexpected results. Contrary to previous reports with glass [11, 14], addition of BSF resulted in an increase in the friction coefficient (Fig. 5c). The same behavior was observed for AGP and BSA (Fig. 5a, b). In this case, the system involves a hydrophobic surface sliding against a hydrophilic one. The absence of chemical interactions between such surfaces generally leads to low friction coefficients in water or saline solutions. The increase in friction caused by BSA could be due to the formation of high-shear-strength layers upon adsorption. The increase caused by AGP is most likely related to its low and slow adsorption onto a hydrophilic surface, as shown by the OWLS results (Fig. 3), meaning that the clean glass surface is only sparsely coated with AGP. There will then be complex electrostatic bridging interactions between the charges on the glycoproteins adsorbed to the latex surface and the exposed glass surface.

The increase of friction caused by addition of BSF can be attributed to contributions from a number of proteins since adsorption appears to be relatively unselective. The relative amounts and conformations of the adsorbed molecules will inevitably play a role.

To determine whether contaminated surfaces, due to inadequate cleaning procedures, could influence protein adsorption to a sufficient extent to cause a change in the friction behavior, ethanol-cleaned glass was used. In previous related studies, cleaning of the glass slides was carried out by means of an ethanol-based detergent [5, 14]. The ethanol-cleaned glass substrates used in this study had a static contact angle of $60^{\circ}$, indicating that the surface was not as clean as the Piranha-cleaned samples. A substantially higher level of hydrophobicity was achieved by functionalizing the glass surface, resulting in a static contact angle of $100^{\circ}$. Friction coefficients in both cases were higher in PBS than for the Piranha-cleaned glass, presumably due to hydrophobic interactions between the contacting surfaces. A decrease in the friction coefficient was observed upon addition of BSF and AGP, as reported by others for BSF $[11,14]$. AGP adsorbs faster and to a greater extent on hydrophobic surfaces, as shown by the OWLS measurements and fluorescence studies presented in previous work, forming a packed layer determined by the size and packing of the glycan residues [18]. Its good lubricating properties could, therefore, be attributed to the presence of glycans on both contacting surfaces, providing a highly hydrophilic layer capable of trapping water at the surface to provide a lubricious, low-shear-strength liquid film. The ethanol-cleaned glass, with a static contact angle of $60^{\circ}$, displayed similar behavior to that of the hydrophobized glass with a static contact angle of $100^{\circ}$. This suggests that the proteins interact with the ethanol-cleaned glass through hydrophobic interactions and demonstrates the importance of the cleaning procedure. BSA impaired lubrication upon its addition to PBS for latex sliding against both hydrophilic glass and hydrophobic glass. The denaturation of BSA upon adsorption on a hydrophobic surface results in a high-shear-strength layer with strong hydrophobic interactions between the albumin and the surface and between adjacent albumin molecules, which can account for the poor lubricating properties [19].

Similarly to AGP, BSF improved the lubrication upon addition to PBS. As albumin is the most abundant component of synovial fluid, it may be expected to give a major 
contribution to the friction. However, other synovial fluid proteins as well as glycoproteins such as AGP and alpha1-antitrypsin (A1AT) have been previously shown to adsorb on both hydrophobic and hydrophilic surfaces [18]; BSA, AGP, and A1AT were all found to adsorb to a greater extent on the hydrophobic surface. Although the adsorption studies showed that all investigated proteins adsorbed to some extent from BSF onto both hydrophobic and hydrophilic surfaces, the influence of surface chemistry on the friction behavior of BSF suggests that some degree of selectivity in the relative amounts of adsorbed proteins may be present. The higher friction coefficient for hydrophilic glass, compared to that for hydrophobic glass, suggests that the proportion of albumin adsorbed, relative to glycoprotein, is higher on hydrophilic surfaces. This is supported by OWLS results that show that BSA adsorbed faster and to a greater extent on a hydrophilic surface in comparison to AGP (Fig. 3). On the other hand, when BSF is used as a lubricant in the latex-versushydrophobic-glass system, more of the glycoproteins, relative to albumin, may be adsorbing on both surfaces. This would then, to some extent, counteract the negative effect of albumin on friction. Synovial fluid is an extremely complex mixture of biomolecules, and therefore many other components or combinations of components may be influencing friction. Additionally, the friction coefficients will likely be not only influenced by the relative adsorption of the different proteins but also by the structure of the adsorbed proteins, which is highly sensitive to the surface chemistry.

\section{Conclusion}

The precise surface chemistry of the sliding surfaces plays an important role in the lubrication of latex sliding against glass, which has been extensively used as a model system for the investigation of natural joint tribology. Surface hydrophobicity, largely determined by the effectiveness of the cleaning procedure, has been demonstrated to exert a strong influence on the lubricating properties of the synovial-fluid proteins investigated. Furthermore, AGP, a glycoprotein present in synovial fluid, was shown to decrease friction in the latex-on-glass system, when cleaned according to methods described in the literature, showing that lubricin is not unique in this regard.

In summary, latex on glass appears to be a poor model for cartilage-cartilage systems, as surface chemistry inevitably plays a role in synovial fluid protein adsorption and lubrication, and literature reports may have been dominated by surface-contamination-induced artifacts.

\section{References}

1. Prete, P.E., Gurakar-Osborne, A., Kashyap, M.L.: Synovial fluid lipids and apolipoproteins: a contemporary perspective. Biorheology 32(1), 1-16 (1995)

2. Foster, H., Fisher, J.: The influence of loading time and lubricant on the friction of articular cartilage. Proc. Inst. Mech. Eng. G 210 , 109-119 (1996)

3. Roberts, B.J., Unsworth, A., Mian, N.: Modes of lubrication in human hip joints. Ann. Rheum. Dis. 41, 217-224 (1982)

4. Crockett, R.: Boundary lubrication in natural articular joints. Tribol. Lett. 35(2), 77-84 (2009)

5. Jay, G.D., Tantravahi, U., Britt, D.E., Barrach, H.J., Cha, C.-J.: Homology of lubricin and superficial zone protein (SZP): products of megakaryocyte stimulating factor (MSF) gene expression by human synovial fibroblasts and articular chondrocytes localized to chromosome 1q25. J. Orthop. Res. 19(4), 677-687 (2001)

6. Swann, D.A., Hendren, R.B., Radin, E.L., Sotman, S.L., Duda, E.A.: The lubricating activity of synovial fluid glycoproteins. Arthritis Rheum. 24(1), 22-30 (1981)

7. Swann, D.A., Radin, E.L.: The molecular basis of articular joint lubrication. 1. Purification and properties of a lubricating fraction from bovine synovial fluid. J. Biol. Chem. 247(24), 8069-8073 (1972)

8. Radin, E.L., Swann, D.A., Weisser, P.A.: Separation of a hyaluronate-free lubricating fraction from synovial fluid. Nature 228(5269), 377-378 (1970)

9. Crockett, R., Grubelnik, A., Roos, S., Dora, C., Born, W., Troxler, H.: Biochemical composition of the superficial layer of articular cartilage. J. Biomed. Mater. Res. A 82A(4), 958-964 (2007)

10. Crockett, R., Roos, S., Rossbach, P., Dora, C., Born, W., Troxler, H.: Imaging of the surface of human and bovine articular cartilage with ESEM and AFM. Tribol. Lett. 19(4), 311-317 (2005)

11. Davis Jr., W.H., Lee, S.L., Sokoloff, L.: Boundary lubricating ability of synovial fluid in degenerative joint disease. Arthritis Rheum. 21(7), 754-760 (1978)

12. Jay, G.D.: Characterization of a bovine synovial fluid lubricating factor. 1. Chemical, surface activity and lubricating properties. Connect. Tissue Res. 28, 71-88 (1992)

13. Jay, G.D., Britt, D.E., Cha, C.J.: Lubricin is a product of megakaryocyte stimulating factor gene expression by human synovial fibroblasts. J. Rheumatol. 27, 594-600 (2000)

14. Jay, G.D., Haberstroh, K., Cha, C.J.: Comparison of the boundary-lubricating ability of bovine synovial fluid, lubricin, and Healon. J. Biomed. Mater. Res. 40(3), 414-418 (1998)

15. Chang, D.P., Abu-Lail, N.I., Guilak, F., Jay, G.D., Zauscher, S.: Conformational mechanics, adsorption, and normal force interactions of lubricin and hyaluronic acid on model surfaces. Langmuir 24(4), 1183-1193 (2008)

16. Zappone, B., Ruths, M., Greene, G.W., Jay, G.D., Israelachvili, J.N.: Adsorption, lubrication and wear of lubricin on model surfaces: polymer brush-like behavior of a glycoprotein. Biophys. J. 92, 1693-1708 (2007)

17. Raviv, U., Giasson, S., Kampf, N., Gohy, J.-F., Jerome, R., Klein, J.: Lubrication by charged polymers. Nature 425(6954), 163-165 (2003)

18. Roba, M., Naka, M., Gautier, E., Spencer, N.D., Crockett, R.: The adsorption and lubrication behavior of synovial fluid proteins and glycoproteins on the bearing-surface materials of hip replacements. Biomaterials 30(11), 2072-2078 (2009)

19. Heuberger, M.P., Widmer, M.R., Zobeley, E., Glockshuber, R., Spencer, N.D.: Protein-mediated boundary lubrication in arthroplasty. Biomaterials 26(10), 1165-1173 (2005) 